\title{
Remoción de cromo en efluente de curtiembre por consorcio de levaduras del género Saccharomyces y Pichia
}

\author{
RODRÍGUEZ YUPANQUI, Magda”; QUEZADA ALVAREZ Medardo Alberto²,
}

\section{RESUMEN}

El efluente del proceso del curtido genera impactos negativos en la salud y el ambiente debido a que en esta etapa solo se aprovecha el $70 \%$ del cromo $(\mathrm{Cr})$ total utilizado; por lo cual la bioadsorción surge como una alternativa en la remoción de metales pesados. En tal sentido, el objetivo de la presente investigación fue evaluar la capacidad de remoción de cromo en efluentes de curtiembre utilizando un consorcio de levaduras constituido por cepas de Saccharomyces cerevisiae (S) y Pichia guilliermondi $(P)$ aislados de residuos agroindustriales. El diseño experimental consistió en 4 biorreactores de $250 \mathrm{ml}$ condicionados con muestras de efluente de la etapa de curtido, teniendo como sorbente el consorcio ( $\mathrm{S}+\mathrm{P}$ ), controlados a 0 , 6, 12 y 24 horas. Las muestras fueron analizadas por la técnica de espectrofotometría de absorción atómica a la flama. Los resultados muestran una capacidad de remoción de Cr total de $57 \%$ y $54 \%$ en concentración de 50 y 100 ppm respectivamente por el consorcio de levaduras; asimismo, la evaluación estadística con ANOVA permite afirmar que no existe diferencia significativa $(p>0.05)$ al emplear ambas concentraciones, recomendándose el uso de la más alta en el proceso de bioadsorción de efluentes de curtiembres.

Palabras clave: Curtido, remoción, cromo, levaduras y consorcio.

\section{Removal of chrome in tannery effluent by consortium of yeasts of the genus Saccharomyces and Pichia}

\begin{abstract}
The effluent from the tanning process generates negative impacts on health and the environment since only $70 \%$ of the total chrome ( $\mathrm{Cr}$ ) used is exploited at this stage; Therefore, bioabsorption emerges as an alternative in the removal of heavy metals. In that sense, the objective of the present investigation was to evaluate the chrome removal capacity in tannery effluents using a consortium of yeasts composed of Saccharomyces cerevisiae (S) and Pichia guilliermondi (P) strains isolated from agro-industrial waste. The experimental design consisted of 4 bioreactors of $250 \mathrm{ml}$ conditioned with samples of effluent from the tanning stage, having the consortium $(\mathrm{S}+\mathrm{P})$ as sorbent, and controlled at $0,6,12$ and 24 hours. The samples were analyzed by the flame atomic absorption spectrophotometry technique. The results show a total $\mathrm{Cr}$ removal capacity of $57 \%$ and $54 \%$ in concentrations of 50 and $100 \mathrm{ppm}$ respectively by the yeast consortium; likewise, the statistical evaluation with ANOVA allows affirming that there is no significant difference $(p>0.05)$ when using both concentrations, recommending the use of the higher one in the bioabsorption process of tannery effluents.
\end{abstract}

Keywords: Tanning, removal, chrome, yeast and consortium

Universidad César Vallejo. Escuela de Ingeniería Ambiental, ORCID:0000-0002-9354-2786, mrodriguez@ucv.edu.pe

2Universidad Nacional de Trujillo. Escuela de Ingeniería Ambiental, ORCID:0000-0002-0215-5175, maqa69@hotmail.com 


\section{INTRODUCCIÓN}

Desde el inicio de la revolución industrial, la humanidad ha estado introduciendo numerosas sustancias peligrosas, en su mayoría metales pesados que son vertidos al ambiente a un ritmo exponencial. La persistente contaminación representa una gran amenaza para todas las formas de vida en el medio ambiente, afectando negativamente el crecimiento de las plantas y la variación genética (Kumar et al., 2017) debido a sus efectos tóxicos (Rubio et al., 2015). Estos metales son muy reactivos a bajas concentraciones y pueden acumularse en la red alimenticia, causando graves problemas en los ecosistemas y la salud pública (Ayangbenro et al., 2017).

El cromo es uno de los metales pesados más tóxico y común entre los contaminantes presentes en los efluentes industriales (Benazir et al., 2010) y está presente en la naturaleza en varios estados de oxidación de $\mathrm{Cr}$ (III) a $\mathrm{Cr}$ (VI). Sin embargo, el $\mathrm{Cr}$ (VI) es una sustancia cancerígena mientras que $\mathrm{Cr}$ (III) es menos tóxico, insoluble y es un nutriente esencial para los humanos (Mishra et al., 2016). Recientemente, la eliminación de metales pesados de las aguas residuales usa varios métodos convencionales, como los químicos mediante precipitación, coagulación-floculación, flotación, intercambio iónico y filtración por membrana; sin embargo, estos métodos no son económicos, utilizan grandes cantidades de productos químicos y generan lodos tóxicos (Siddiquee et al., 2015).

La industria de curtiembres contribuye especialmente a la contaminación por cromo, al consumir aproximadamente el $32 \%$ del cromo total mundial, que al ser vertido al ambiente suele acumularse en los sedimentos y/o transforman el ambiente (Parveen et al., 2017). La recuperación de $\mathrm{Cr}$ a partir de las aguas residuales producidas en la etapa de curtido es una alternativa a favor del ambiente que evita la eliminación de gran cantidad de lodo que contiene $\mathrm{Cr}$ en vertederos de residuos industriales peligrosos (Mella et al., 2015). Además, las personas que participan del proceso productivo del cuero tienen un riesgo significativo a la exposición a cromo (Cuberos et al., 2019).

Por lo tanto, existe una creciente necesidad de desarrollar una nueva propuesta ecológica y rentable para la remediación de metales inorgánicos $(\mathrm{Cr}, \mathrm{Hg}, \mathrm{Cd}$ y $\mathrm{Pb}$ ) antes de ser liberados al ambiente y salvaguardar el ecosistema (Marzan et al., 2017; Igiri et al.,
2018). La biorremediación es un método de tratamiento biológico, que requiere de organismos vivos como plantas y microorganismos, (bacterias y hongos) que destruyen y/o transforman contaminantes peligrosos (Maier et al., 2009). Estos microorganismos tienen diversos mecanismos de secuestro de metales o tienen mayores capacidades de biosorción de metales, utilizando biomasa viva o muerta y sus componentes como las enzimas (Benazir et al., 2010). Este método ofrece una alta especificidad mediante mecanismos de bioadsorción, biodisponibilidad bioacumulación, biotransformación o biolixiviación (Fang et al., 2007 y Dzionek et al., 2016). Además, la capacidad de biosorción aplicando bacterias, hongos, biopelículas, algas, microorganismos genéticamente modificados $y$ células microbianas inmovilizadas y biofilm ha demostrado tener un efecto sinérgico en la eliminación de los metales pesados (Tejada et al., 2015; Igiri et al., 2018).

La biosorción de iones metálicos tiene lugar en la superficie externa de la pared celular de los microorganismos. Este proceso proporciona un mecanismo para inmovilizar metales pesados tóxicos y evitar una mayor entrada en las células vivas que podría causar daño significativo a las enzimas funcionales. La adsorción es un proceso físico que se da a través de las fuerzas electrostáticas o de van der Waals que retienen los iones metálicos que son adsorbidos, uniendo grupos químicos funcionales de las biomoléculas en la superficie celular y luego puede atravesar la estructura celular a través de interacciones químicas (Han, X., \& Gu, J. D., 2010). Se han realizado estudios con hongos sobre los mecanismos de interacción con el cromo, mediante procesos de biosorción y de bioacumulación. Las especies reportadas incluyen levaduras como Saccharomyces cerevisiae, Candida sp., Pichia sp. y los hongos filamentos (Benitez-Campos, 2011).Estudios recientes, han descrito cepas fúngicas capaces de realizar el proceso de transformación de $\mathrm{Cr}$ (VI) a especies reducidas.

Varios autores vienen investigando la remediación de cromo con levaduras del genero Saccharomyces (Wu et al., 2011 y Santoyo, 2017); otros investigadores como Dimas et al. (2013) reporta la interacción de Cr III con otros metales pesados en el proceso de biosorción de levadura de pan (Saccharomyces cerevisiae) demostrando una mayor capacidad de remoción con estas levaduras; Amils et al. (2004), sostiene 
que los hongos se pueden desarrollar en medio ácido y en presencia de cromo, y después adaptación al medio son capaces de secuestrarlo e eliminarlo del medio; Orbegozo et al. (2008), analiza la presencia de levaduras en aguas ácidas de minas, entre ellos hongos y protistas; y sostiene que Pichia guillermondi, presentan una alta resistencia a metales pesados; Ksheminska et al. (2003), reporta que la mayoría de estas cepas naturales de levaduras son más tolerantes al cromo y capaces de crecer en presencia de $\mathrm{Cr}$ III a 5 mM o Cr VI a 0.5 mM; en el año 2005 Ksheminska et al., describe que tanto levaduras como hongos filamentosos son capaces de acumular concentraciones elevadas de cromo en la biomasa; como ejemplo, indica que la levadura $P$. guillermondii puede acumular alrededor de $13.0 \mathrm{mg}$ de $\mathrm{Cr} / \mathrm{g}$ de biomasa más que las hongos filamentosos; Fernandez et al. (2012) analizan la resistencia de cepas indígenas de $P$. jadinii M9 y $P$. anomala M10, a altas concentraciones de $\mathrm{Cr}$ VI.

Saccharomyces cerevisiae, es una levadura que constituye el grupo de microorganismos eucariotas, unicelulares presenta células redondas a ovoides, de 5 a $10 \mu \mathrm{m}$ de diámetro, se reproducen en forma asexual (brotación o gemación y división transversal) y sexual (por esporas), presentan hifas; sus principales constituyentes macromoleculares según Suárez (2016) incluyen proteínas, glicoproteínas, polisacáridos, polifosfatos, lípidos y ácidos nucleicos, su pared celular contiene entre 15 y 25 $\%$ de la masa seca de la célula y está compuesta de polisacáridos $(80-90 \%)$, esencialmente glucanos y mananos, con una menor contribución de quitina, además de proteínas y lípidos. Sin embargo, Pichia guilliermondii o Meyerozyma guilliermondii es una levadura ampliamente distribuida en el medio ambiente natural, es de fácil aislamiento a partir de frutas y bayas, con alto contenido de azúcar (Rosales, 2018), las células de $P$. guilliermondii son heterogéneos, en su mayoría de forma alargada (mide aprox. $20 \mu \mathrm{m}$ ) a veces forma un pseudomicelio, crece a $30^{\circ} \mathrm{C}$ hasta puede llegar hasta $42^{\circ} \mathrm{C}$ (Sibirny et al., 2009).

El uso de consorcios microbianos para degradar contaminantes es una tendencia importante para fines de la biorremediaciòn por su alta eficiencia en la degradación (Macchi, 2018; Centeno et al., 2019). El tratamiento biológico de aguas contaminadas, mediante consorcios microbianos autóctonos, aprovecha las potencialidades metabólicas de grupos distintos de microorganismos para degradar y/o remover una amplia variedad de compuestos (Díaz et al., 2018). Se han realizado estudios aplicando consorcios microbianos como Bhattacharya et al. (2017) utilizo un consorcio de cuatro cepas bacterianas en los efluentes de curtiembre $(\mathrm{pH}$ $4,6)$ logrando eliminar el $78 \%$ de $\mathrm{Cr}(\mathrm{VI})$, a las 96 $\mathrm{h}$ de tratamiento. En general se puede inferir que el consorcio bacteriano podría ser utilizado efectivamente para el tratamiento $\mathrm{Cr}$ (VI) en la curtiembre y otros efluentes industriales.

Por tal motivo la presente investigación tiene como objetivo, evaluar la capacidad de remoción de cromo aplicando un consorcio microbiano de levaduras de Saccharomyces cerevisiae y Pichia guilliermondi en efluente de la industria de curtiembre.

\section{MATERIAL Y MÉTODOS}

\subsection{MATERIALES}

El consorcio microbiano pertenece a las cepas de levaduras de Saccharomyces cerevisiae aisladas del jugo de caña de azúcar (Saccharum officinarum) y cepas de levaduras de Pichia guilliermondii aisladas del jugo de uva (Vitis vinifera) cuya finalidad es actuar como sorbente del $\mathrm{Cr}$ total presente en el efluente.

El efluente recolectado fue agua residual de la etapa de curtido proveniente de la curtiembre INVERSIONES HAROD S.A.C., ubicado en Mz. C3 Lote. 01, Parque Industrial, distrito de La Esperanza, es el material de estudio el cual va a ser expuesto al consorcio microbiano de levaduras.

\subsection{PROCEDIMIENTO}

\subsubsection{OBTENCIÓN DE BIOMASA DEL CONSORCIO MICROBIANO DEL GÉNERO SACCHAROMYCES Y PICHIA}

Del cultivo puro de Saccharomyces cerevisiae y Pichia guilliermondii se realizó una suspensión para ser cultivada en medio liquido de caldo Sabouraud a un pH 5,0 (Ksheminska et al., 2005). Se utilizarón 4 matraces de $250 \mathrm{ml}$, luego se incubo en un Shaking Incubador LBSI-100A a $30^{\circ} \mathrm{C}$ con una agitación de $200 \mathrm{rpm}$ por 24 horas, transcurrido el tiempo de incubación se procedió a cosechar las células de levaduras. Se procedió a centrifugar por 5 minutos utilizando una Centrifuga Universal Mo. PLC-012E con tubos de 
falcón de $50 \mathrm{ml}$ para facilitar el proceso de centrifugado y se lavó varias veces con agua desionizada (Wu et al., 2011). Seguidamente se separó el sobrenadante y se pesó la biomasa con una balanza Ohaus Harvard Trip Balance Mo. 1450-SD hasta obtener $1.0 \mathrm{~g}$ de biomasa de Saccharomyces cerevisiae. Del mismo modo se cultivó y cosecho biomasa de Pichia guilliermondii obteniendo la misma cantidad. La biomasa se almacenó a temperatura de refrigeración $\left(4^{\circ} \mathrm{C}\right)$ para los siguientes experimentos.

\subsubsection{ACONDICIONAMIENTODE BIORREACTORES}

Según el diseño experimental se utilizó 04 biorreactores, matraz Erlenmeyer marca pyrex de capacidad de 250 ml previamente esterilizados taponados con algodón.

\section{2. 3 R E C O L E C C I Ó N $\mathbf{N}$ ACONDICIONAMIENTO DE EFLUENTE DE CURTIEMBRE}

Las muestras del efluente (aguas residuales de la etapa de curtido) fueron recolectas en botellas de vidrio estériles de $2 L$ de capacidad debidamente etiquetadas. Posteriormente fueron transportadas al Laboratorio de Biotecnología de la Escuela de Ingeniería Ambiental de la UCV - CIT MOCHE para su análisis fisicoquímico. En seguida se determinó el $\mathrm{pH}$ inicial de 3,45 (pH-metro de Mesa HANNA INSTRUMENTS- Mo. HI2213), la turbidez fue de 12,6 NTU (Turbidímetro, HANNA INSTRUMENTSMo. HI83414) y la temperatura del efluente fue de $26,9^{\circ} \mathrm{C}$ (Termómetro de mercurio, marca BOECO).

Luego se determinó la concentración de Cr total presente en la muestra del efluente de la etapa de curtido, reportando un valor de 964,074 ppm aplicando la técnica de espectrofotometría de absorción atómica a la flama (Espectrofotómetro de absorción atómica, marca PG Instruments, Mo. AA5000FG con un software AAWin3).

\subsubsection{MONITOREO Y EVALUACIÓN DEL PROCESO DE BIOSORCIÓN}

Se trabajó con 04 biorreactores de capacidad de
$250 \mathrm{ml}$; primero, se adicionó $150 \mathrm{ml}$ de muestra de efluente de curtiembre a una concentración de Cr total de 50 ppm en dos matraces y se agregó $1 \mathrm{~g}$ de consorcio de levaduras $(P+S)$; seguidamente se realizó la misma técnica a otros dos matraces agregando $150 \mathrm{ml}$ de muestra de efluente a una concentración de 100 ppm de Cr total y se adiciono $1 \mathrm{~g}$ de consorcio de levadura, utilizando la metodología de Krauter et al., (1996). Los biorreactores se incubaron en un Shaking Incubador por 24 horas, a $30^{\circ} \mathrm{C}$ y $200 \mathrm{rpm}$ de agitación. El pH se condiciono a 5 según Salari \& Salari (2017).

Se tomaron muestras por triplicado a las $0,6,12$ y 24 horas, en cada periodo se tomó $10 \mathrm{ml}$ y se procedió a centrifugar; luego se separó el sobrenadante y se procedió a filtrar utilizando un Syringe Filters Nylon de $0.45 \mu m$ (Ferraz, 2004) con diámetro de $13 \mathrm{~mm}$ para separar posible microbiomasa, se almaceno por triplicado el sobrenadante en 3 microtubos de $2 \mathrm{ml}$. Se procedió a codificar las muestras para sus respectivos análisis de cromo.

\subsubsection{DETERMINACIÓN DE LOS NIVELES DE CROMO POR ABSORCIÓN ATÓMICA (AA)}

La determinación de $\mathrm{Cr}$ total se realizó mediante el método de Espectrofotometría de absorción atómica a la flama, teniendo en cuenta los estándares de APHA (3111B -Método directo de llama aire-acetileno), AWWA (3111A edición 21, 2005), WPCF (1985) y el manual de métodos analíticos para espectrofotometría de Absorción Atómica de PERKIN ELMER (1982)

\subsection{MÉTODO}

El análisis del diseño experimental fue unifactorial teniendo como Variable Dependiente la Capacidad de adsorción de cromo (Porcentaje) y la Variable Independiente el Consorcio microbiano de levaduras (g. Saccharomyces + g. Pichia) ver Tabla 1. 
Tabla 1. Análisis del diseño experimental

\begin{tabular}{|c|c|c|c|c|c|c|c|}
\hline \multirow{2}{*}{$\begin{array}{l}\text { Tipo de } \\
\text { biomasa }\end{array}$} & \multirow{2}{*}{$\begin{array}{c}\text { Concentration } \\
\text { de } \mathrm{Cr}\end{array}$} & \multirow{2}{*}{$\begin{array}{c}\text { Peso } \\
\text { Biomasa }\end{array}$} & \multicolumn{4}{|c|}{ Tiempo } & \multirow{2}{*}{$\begin{array}{l}\text { Rendimiento } \\
\text { Promedio }\end{array}$} \\
\hline & & & Oh & $6 h$ & $12 \mathrm{~h}$ & $24 h$ & \\
\hline \multirow{3}{*}{$\mathrm{S}+\mathrm{P}$} & \multirow{3}{*}{$50 \mathrm{ppm}$} & \multirow{3}{*}{$1 \mathrm{~g}$} & $x$ & $x x$ & $x \times x$ & $x \times x x$ & IV \\
\hline & & & $x$ & $x x$ & $x \times x$ & $x \times x x$ & IV \\
\hline & & & $x$ & $x x$ & $x \times x$ & $x \times x x$ & IV \\
\hline \multirow{3}{*}{$\begin{array}{c}\text { Consorcio } \\
\text { de } \\
\text { levaduras }\end{array}$} & \multirow{3}{*}{$100 \mathrm{ppm}$} & \multirow{3}{*}{$1 \mathrm{~g}$} & $x$ & $x x$ & $x \times x$ & $x \times x x$ & IV \\
\hline & & & $\mathrm{x}$ & $x x$ & $x \times x$ & $x \times x x$ & IV \\
\hline & & & $x$ & $x x$ & $x \times x$ & $x \times x x$ & IV \\
\hline
\end{tabular}

Se realizó un análisis de varianza (ANOVA) para estimar diferencias significativas entre el consorcio y la concentración de cromo total a un nivel de confianza de 95\%. Estos análisis se realizaron utilizando el programa IBM-SPSS Statistics 22.0 para Windows.

\section{RESULTADOS}

En el presente trabajo de investigación se tiene como referencia la evaluación del efluente de la etapa de curtido de la empresa curtidora Inversiones Harold SAC, tal como se reporta en la Tabla 2. Se puede observar las características fisicoquímicas (Parámetros) del efluente en especial el valor de $\mathrm{Cr}$ total es un referente de la necesidad de realizar un tratamiento previo antes de ser vertido al ambiente.

Tabla 2. Características fisicoquímicas del efluente de la etapa de curtido de la curtiembre Inversiones Harold S.A.C.

\begin{tabular}{ccc} 
Parámetros & Valor & $\begin{array}{c}\text { DS. 0:2015 } \\
\text { Vivienda }\end{array}$ \\
\hline T०C $^{\circ}$ & 26,9 & $<35$ \\
\hline pH & 3,45 & $6-9$ \\
\hline $\begin{array}{c}\text { Turbidez (NTU) } \\
\text { Crtotal } \\
\text { ppm }\end{array}$ & 1,6 & 8,5 \\
\hline
\end{tabular}

En la Figura 1, se observa el porcentaje de remoción de $\mathrm{Cr}$ total durante 24 horas utilizando un consorcio microbiano de levadura de Saccharomyces Cerevisiae y Pichia guilliermondii y aplicando dos tratamientos en concentración de 50 y 100 ppm. Dicha grafica muestra a las 6 horas un porcentaje de remoción de valores similares entre $39,36 \%$ y $40,54 \%$ respectivamente; sin embargo, a partir de las 12 y 24 horas se observa una diferencia de $8,17 \%$ en ambas concentraciones y luego se reporta un valor de $3,49 \%$ de diferencia de remoción de cromo siendo la más alta a 50 ppm y el menor valor a 100 ppm respectivamente.

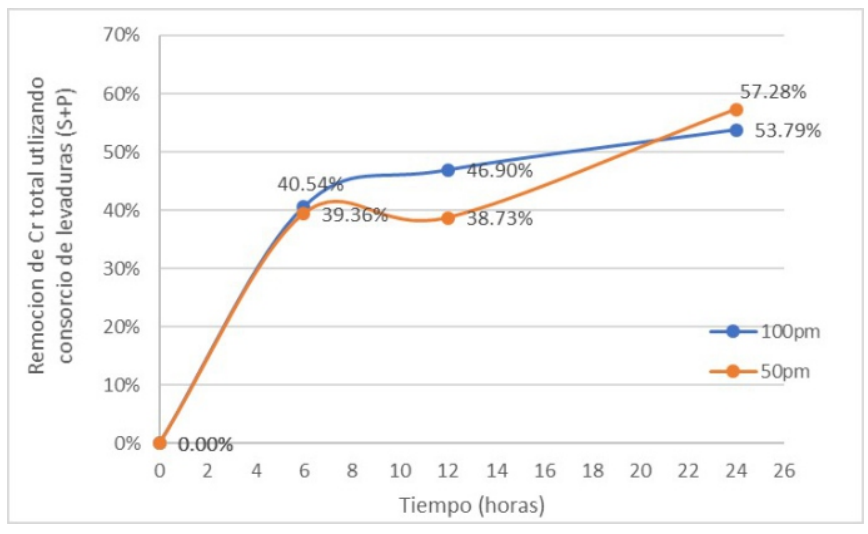

Figura 1: Porcentaje de remoción de Cr total en función al tiempo aplicando un consorcio de levaduras en concentración de 50 y 100 ppm de cromo. 
En la Figura 2, se observa la cantidad $\mathrm{Cr}$ total residual del efluente de la curtiembre en relación a la concentración de 50 y 100 ppm aplicando un consorcio de levaduras de Saccharomyces cerevisiae y Pichia guilliermondii en 6, 12 y 24 horas. Según la gráfica existe una tendencia de disminuir el $\mathrm{Cr}$ total residual a una concentración inicial de $100 \mathrm{ppm}$ se reporta durante las primeras
6 horas de 61,7 ppm; a las 12 horas 55,1 ppm y a las 24 horas 47,9 ppm. Luego se observó que la cantidad de $\mathrm{Cr}$ total residual a una concentración de 50 ppm disminuye en 28,7 ppm a las 6 horas, pero a las 12 horas sufre un ligero incremento de 29,0 ppm y por último a las 24 horas llega a 20,2 ppm.

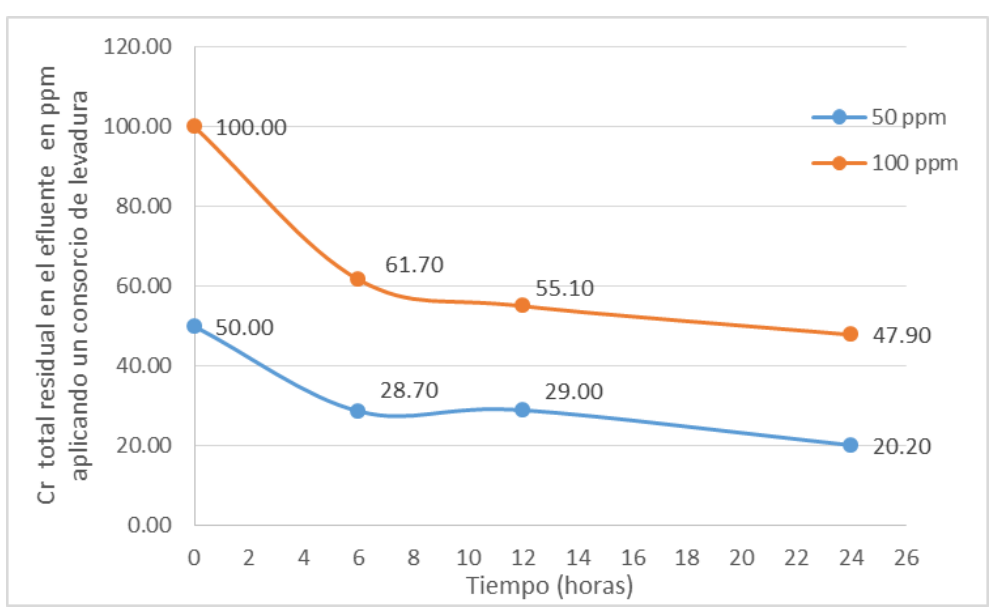

Figura 2: Porcentaje de remoción de Cr total en función al tiempo aplicando un consorcio de levaduras en concentración de 50 y 100 ppm de cromo.

En la Figura 3, se observa que el color del efluente tiene un color verdoso como características de la aplicación de óxido de cromo en la etapa de curtido. Después de agregar la biomasa de consorcio de levadura se torna un color verde lechoso y va cambiando en función al tiempo más claro.

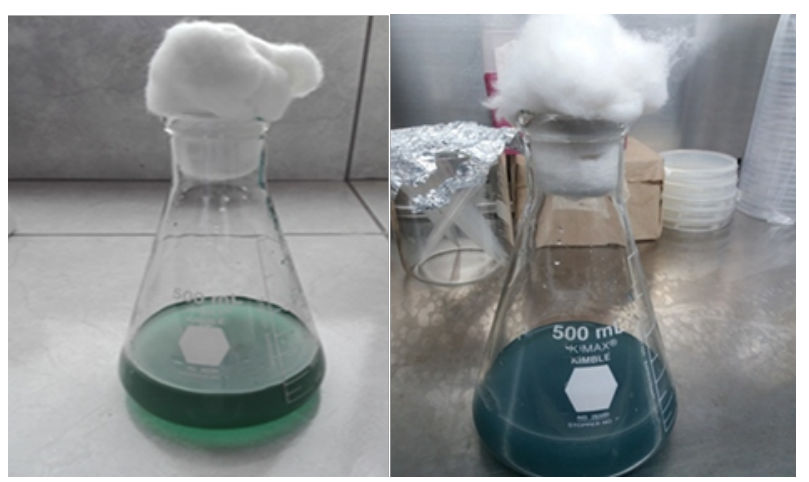

Figura 3: Color del efluente de la curtiembre antes y después del tratamiento con biomasa de consorcio de levaduras.

En la tabla $\mathrm{N}^{\circ} 3$, se puede observar del análisis de las tres repeticiones de los resultados del $\mathrm{Cr}$ total del efluente se evidencia el promedio según el reporte de la Media, se puede evidenciar una diferencia entre las dos concentraciones. Además, se puede observar el valor mínimo de
54,19 de $\mathrm{Cr}$ total y el máximo de 59,75 a una concentración de $50 \mathrm{ppm}$. También se puede observar el valor mínimo de 52,65 y el valor máximo de 54,35 a una concentración de 100 ppm. 
Tabla 3. Medias del porcentaje de remoción de cromo total a 50 y 100 ppm de Cr, con $1 \mathrm{~g}$ de biomasa del consorcio de levaduras durante 24 horas y nível de confianza de $95 \%$

\begin{tabular}{|c|c|c|c|c|c|c|c|c|}
\hline & \multicolumn{7}{|c|}{ Porcentaje de remoción de cromo en 24 horas } & \multirow{3}{*}{ Máximo } \\
\hline & \multirow[t]{2}{*}{$\mathrm{N}$} & \multirow[t]{2}{*}{ M } & \multirow[t]{2}{*}{ DE } & \multirow[t]{2}{*}{ ESD } & \multicolumn{2}{|c|}{$\begin{array}{c}95 \% \text { del intervalo } \\
\text { de confianza para } \\
\text { la media }\end{array}$} & \multirow[t]{2}{*}{ Mínimo } & \\
\hline & & & & & LI & LS & & \\
\hline $\begin{array}{c}50 \\
\mathrm{ppm}\end{array}$ & 3 & 57,28 & 2,83 & 1,63 & 50,24 & 64,32 & 54,19 & 59,75 \\
\hline $\begin{array}{l}100 \\
\text { ppm }\end{array}$ & 3 & 53,78 & 0,98 & 0,56 & 51,34 & 56,22 & 52,65 & 54,35 \\
\hline Total & 6 & 55,53 & 2,69 & 1,10 & 52,70 & 58,36 & 52,65 & 59,75 \\
\hline
\end{tabular}

En la Tabla 4, se describe el análisis de Varianza Entre grupos, conformado por las concentraciones de 50 y $100 \mathrm{ppm}$ empleadas.
Tambien se tiene el análisis Dentro de grupos referido a las tres observaciones de cada grupo tanto para 50 y $100 \mathrm{ppm}$.

Tabla 4. Análisis de varianza a 50 y 100 ppm de cromo, con biomasa del consorcio durante 24 horas, com nível de significancia de $p>0,05$

\begin{tabular}{cccccc}
\hline \multicolumn{6}{c}{ Análisis de Varianza } \\
\hline \multicolumn{7}{c}{ Porcentaje de remoción de cromo $(24$ horas) } \\
\hline SC & gl & MC & F & Sig.* \\
\hline $\begin{array}{c}\text { Entre grupo } \\
\text { Dentro de } \\
\text { grupos }\end{array}$ & 18,37 & 1 & 18,37 & 4,08 & 0,113 \\
\hline Total & 17,98 & 4 & 4,49 & & \\
\hline
\end{tabular}

*No significativo

\section{DISCUSIÓN}

Las características fisicoquímicas del efluente de la etapa de curtido se muestran en la tabla 2. Los parámetros físicos del efluente evaluados como To, pH y Turbidez estuvieron por debajo de lo admitido según la norma técnica peruana; sin embargo, la concentración de $\mathrm{Cr}$ total se encontraron valores muy altos de lo prescrito en el DS. 01-2015 Vivienda, donde menciona los valores máximo admisible (VMA) de efluentes no domestico para ser vertido al alcantarillado y cabe mencionar que el reporte obtenido de la cantidad de $\mathrm{Cr}$ total supera los 10ppm de los VMA y de esta manera podemos decir que la contaminación producida por las curtiembre es muy alto en relación al $\mathrm{Cr}$ total concordando con Benítez-Campos, 2013 y Parveen y Singh, 2017 y además Bhattacharya et al., 2015 y Mella et al., 2015, sostienen que el efluente de curtiembre contiene una gran cantidad de contaminantes que incluyen materia orgánica, compuestos fenólicos, taninos y cromo y genera grandes impactos ambientales que amenaza el ambiente. Por lo tanto, se debe dar un tratamiento de las aguas residuales de curtiembre antes de ser vertido al ambiente para evitar su alteración. El presente estudio evalúa la capacidad de remoción de cromo del efluente de la etapa de curtido aplicando un consorcio microbiano constituido por levaduras del género Saccharomyces tal como lo reporta los estudios de Wang et al., (2009); Wu et al., (2011) y Santoyo, (2017) y el género Pichia fue estudiado por Orbegozo et al., (2008); Ksheminska et al., (2005) y Fernández et al., (2012). Dichas cepas, fueron aisladas de muestras residuales agroindustriales como jugo de caña de azúcar y de jugo de uva. Los microorganismos como bacterias y hongos juegan un rol vital en la transformación de elementos traza incluidos los metales pesados como cromo ya que puede influir en su biodisponibilidad y remediación, toxicidad, solubilidad en agua y la movilidad de los metales pesados (Beltrán-Pineda y Gómez-Rodríguez, 2016), pues estos microorganismos cuentan con mecanismos enzimáticos y no enzimáticos para remover metales en solución. Al trabajar con 
consorcio de levaduras del género Saccharomyces y Pichia, se condicionó el medio aplicando un $\mathrm{pH}$ de 5 tal como lo reporta Uscanga, 2005 y Suarez-Machin, et al., 2016 que indica que las levaduras les resulta favorable un medio ligeramente ácido entre 4,5 a 6,5 de pH lo que facilito nuestro trabajo experimental, además el pH es un parámetro importante que afecta la química del metal y sitios de unión de metal en la superficie celular reportado por Wu et al (2011), logrando una capacidad máxima de remoción con Saccharomyces de $55 \%$ a un $\mathrm{pH}$ de 5 en una concentración de 20 ppm de $\mathrm{Cr}$ (VI) aplicando una temperatura de $30^{\circ} \mathrm{C}$. La figura 1 , indica el porcentaje de remoción de $\mathrm{Cr}$ total utilizando muestras de efluentes de la etapa de curtido, aplicando un consorcio de levaduras de la familia Saccharomycetaceae constituido por Saccharomyces Cerevisiae y Pichia guilliermondii, sometido a tratamiento de concentración de 50 y 100 ppm de $\mathrm{Cr}$ total. Se puede observar, una capacidad de remoción de $57,28 \%$ al ser sometido a una concentración de 50 ppm y se obtuvo un 53,79\% a una concentración de 100 ppm, durante 24 horas aplicando un consorcio microbiano de levaduras. También se puede afirmar que la capacidad de remoción a las 6 horas a una concentración de 50 y $100 \mathrm{ppm}$ se alcanza valores similares de $39,36 \%$ y 40,54 respectivamente. Luego a las 12 horas la capacidad de remoción del consorcio sometido a $50 \mathrm{ppm}$ disminuye a una proporción de $0,17 \%$ en comparación con 100ppm, pero luego llega a superarla a las 24horas. Según Beltrán-Pineda y Gómez-Rodríguez (2016), argumentan que la capacidad de remoción de metales pesados por hongos puede ser superior a la reportada con métodos fisicoquímicos convencionales y que esta capacidad de remoción de los metales pesados por métodos biológicos como la biorremediación puede ser selectiva teniendo en cuenta la capacidad metabólica de cada especie biorremediadora y el tipo de metal. Sin embargo, Tejada-Tovar et al. (2015) argumenta que la bioadsorción es un proceso fisicoquímico que incluye los fenómenos de adsorción y absorción de moléculas y iones y para que sea eficaz este proceso debe existir una gran afinidad entre los grupos funcionales de la biomasa y el contaminante, ya que este último debe ser atraído hacia el sólido y enlazado por diferentes mecanismos (Han, X., \& Gu, J. D., 2010). Además, Benazir et al, (2010), reportan la eficiencia de la $\mathrm{S}$. cereviceae en trabajar en consorcio para remover metales pesados. Durante nuestro experimento se ha controlado la temperatura promedio de $30^{\circ} \mathrm{C}$ y la velocidad de agitación de 200 rpm; según Ayangbenro y
Babalola (2017) y Igiri et al. (2018), reportan que la temperatura juega un papel importante en la adsorción de metales pesados aumentando la velocidad de difusión del adsorbato a través de la pared celular, la solubilidad de los metales y mejora la biodisponibilidad de estos. Además, sostiene que la temperatura de $25^{\circ}$ a $40^{\circ} \mathrm{C}$ puede aumentar la capacidad de adsorción de los metales pesados concordando con lo propuesto. El segundo parámetro controlado fue la velocidad de agitación de 200 rpm durante el experimento que permitió aumentar la capacidad de remoción tal como lo reporta Bhattacharya et al. (2015) trabajando en un rango de 250 rpm y MorenoRivas et al. (2016) manifiesta que antes de unir metales en su superficie, las levaduras también las interiorizan. En relación a la fig.2, se observa la cantidad de $\mathrm{Cr}$ residual obtenido del análisis calculado del efluente de la curtiembre aplicando el consorcio microbiano de levaduras a una concentración de 50 ppm se reporta durante las primeras 6 horas valores que disminuyen la concentración inicial a 28,7 ppm y luego a las 12 horas tuvo un comportamiento similar de 29,0 ppm y finalmente disminuyo a 20,2 ppm a las 24 horas; lo mismo sucedió a una concentración de 100 ppm a las 6 horas se reporta 61,7 ppm , luego a las 12 horas disminuye a 55,1 y finalmente a las 24 horas llega a 47,9; eso significa que la capacidad de bioadsorción podría deberse a la presencia de los componentes de la pared celular de las levaduras para atraer a los iones de los metales pesados como cromo, al contenido de la quitina que poseen las levaduras; así mismo también se podría bioacumularse el $\mathrm{Cr}$ debido a una reacción de reducción el $\mathrm{Cr}$ (VI) a $\mathrm{Cr}$ (III) hasta ingresar al citoplasma y llegar a las vacuolas tal como lo reporta Siddiquee et al. (2015) que afirma que una célula microbiana desarrolla resistencia a los metales pesados a través de la excreción de sustancias quelantes de metales, o por un problema en un sistema de transporte particular, que da como resultado una acumulación celular reducida del ion metálico. Sin embargo, existe otro mecanismo de adaptación y resistencia al cromo por algunas especies de levadura tal como describe Irazusta et al., 2018) al condicionar un medio con cromo (VI) con dos tipos de levaduras para analizar la biosíntesis de proteínas u otras sustancias, encontrando que las levaduras tiene una capacidad de respuesta frente al estrés ambiental produciendo más proteínas, metionina sintasa, proteínas de energía y degradación, y proteínas de reducción de óxido y en otras condiciones hasta los puede bioacumular en las vacuolas mediante un transporte activo de gasto de energía, según refiere Beltrán-Pineda y Gómez-Rodríguez (2016). 
Según la fig. 3. Durante el experimento se encontró que las aguas residuales de la curtiembre se decoloran después del tratamiento microbiano para determinar la remoción de $\mathrm{Cr}$ total, tal como han sido reportados por Alluri et al. (2007) utilizando consorcios de microorganismos; Además hay reporte de trabajos con biomasa de levadura de $\mathrm{g}$. Saccharomyces por Wang y Chen, 2009; Wu et al 2011 y Santoyo, 2017 y con biomasa del g. Pichia por Ksheminska et al, (2005) y Fernández et al, (2012).

El estudio fue contrastado estadísticamente para determinar si existían diferencias significativas entre los dos tratamientos a 50 ppm y 100 ppm, tal como se observa en la tabla 3.

En la tabla 4, se aprecia el análisis de varianza y se evidencia que los resultados del porcentaje de remoción de $\mathrm{Cr}$ total no difieren significativamente $(p>0,05$, siendo este 0,113$)$ al emplear concentraciones iniciales de $\mathrm{Cr}$ total de 50 y 100 ppm; por lo cual resulta indistinto trabajar con sistemas que difieren en su concentración inicial; ya que la capacidad de adsorción del consorcio no se ve afectada por dicha variable.

Se demostró que las cepas de levadura de $S$. cerevisiae y $P$. guilliermondii son tolerantes a los metales y podría remediar el $\mathrm{Cr}$ por biosorción o bioacumulación, demostrado en condiciones aeróbicos. Sin embargo, no se reportan estudios relacionados con consorcios microbianos de levaduras y su aplicación puede ser una alternativa viable para el tratamiento de efluentes de las curtiembres en nuestra región la Libertad, pero a la vez se sugiere seguir investigando para determinar todo el proceso de bioadsorción como de bioacumulación de estas levaduras unicelulares de fácil obtención y poco costo en su producción.

\section{CONCLUSIONES}

El consorcio de levaduras (S. cerevisiae y $P$. guilliermondi) tiene una capacidad de remoción de $57 \%$ y $54 \%$ a una concentración de 50 y 100 ppm de $\mathrm{Cr}$ total respectivamente. La capacidad de remoción aplicando un consorcio de levaduras a una concentración de 50 ppm o 100 ppm es indiferente y se puede utilizar la concentración más alta para lograr la mayor capacidad de remoción. La aplicación de consorcios microbiano de levaduras ( $S$. cereviceae y $P$. guilliermondii) constituye una alternativa viable en la biorremediación de efluentes de curtiembre por su costo y su contribución a la conservación del ambiente, por lo cual se recomienda continuar investigando sobre esta tecnología de tratamiento.

\section{REFERENCIAS BIBLIOGRÁFICAS}

Amils, R., Rodríguez, N., Lalueza, J., Rius, A., Ollé, L., Puig, R., \& Font, J. (2004). Aplicaciones biotecnológicas para la recuperación de $\mathrm{Cr}$ (III) a partir de efluentes de curtición. In presentado en XVI Congreso Latinoamericano de Químicos y técnicos de la Industria del Cuero, Buenos Aires. R e c u p e r a d o d e https://aaqtic.org.ar/congresos/buenosaires20 04/pdf/Trabajo02.pdf

Ayangbenro, A., \& Babalola, O. (2017) A new strategy for heavy metal polluted environments: a review of microbial biosorbents. International journal of environmental research and public $\mathrm{h}$ e a l th, 14 ( 1 ), $94 . \mathrm{d} \mathrm{o} \mathrm{i} \mathrm{:}$ https://doi.org/10.3390/ijerph14010094

Benazir, J., Suganthi, R., Rajvel, D., Pooja, M., \& Mathithumilan, B. (2010). Bioremediation of chromium in tannery effluent by microbial consortia. African journal of biotechnology, 9(21), $3140-3143$. R e c u p e ra d o d e : https://www.ajol.info/index.php/ajb/article/view $\angle 80576 / 70819$

Benitez-Campo, N. (2013) Producción limpia y biorremediación para disminución de la contaminación por cromo en la industria de curtiembres. Ambiente y sostenibilidad, 1, 25-31. DOI: https://doi.org/10.25100/ays.v1i1.4335

Bhattacharya, A., Gupta, A., Kaur, A., \& Malik, D. (2015). Simultaneous bioremediation of phenol and $\mathrm{Cr}$ (VI) from tannery wastewater using bacterial consortium. International Journal of Applied Sciences and Biotechnology, 3(1), 50-55. DOI: https://doi.org/10.3126/ijasbt.v3i1.11889

Centeno, L., Quintana, A., \& López, F. (2019). Efecto de un consorcio microbiano en la eficacia del tratamiento de aguas residuales, Trujillo, Perú. 
A rn a I d o a, $26(1), 433-446$. http://dx.doi.org/10.22497/arnaldoa.261.2612 3

Cuberos, E., Rodriguez, A., \& Prieto, E. (2009). Niveles de cromo y alteraciones de salud en una población expuesta a las actividades de curtiembres en Bogotá, Colombia. Revista de salud pública, 11, 278-289. Recuperado de: https://www.scielosp.org/article/rsap/2009.v11 $\underline{\mathrm{n} 2 / 278-289 /}$

Díaz, L., Leal, J., Díaz, A.,Ferrer, J., \& Avendaño, D. (2018). Consorcio microbiano autóctono para el tratamiento de aguas contaminadas con gasoil del puerto de Isla de Toas (Venezuela). Ciencia e Ingeniería Neogranadina, 28(2), 5-27. R e c u p e r a d o d e : https://www.redalyc.org/pdf/911/Resumenes/ Resumen_91158463001_1.pdf

Dimas, L., Miranda, R., Sosa, C., Cantú, M., Rivera, J., Bustos, D., \& Rodríguez, E. (2013). Biosorción $\mathrm{Pb} 2+, \mathrm{Cd} 2+, \mathrm{Zn} 2+\mathrm{y} \mathrm{Cr} 3+$ con levadura de panaderia. Química Hoy, 3(2), 1-4. $\mathrm{R}$ e c u p e r a d o d e : http://eprints.uanl.mx/3347/1/Art1.pdf

Dzionek, A., Wojcieszyska, D., \& Guzik, U. (2016). Natural carriers in bioremediation: A review. Electronic Journal of Biotechnology, $19(5), \quad 28-36$. http://dx.doi.org/10.1016/j.ejbt.2016.07.003

Ferraz, A. I., Tavares, T., \& Teixeira, J. A. (2004). $\mathrm{Cr}$ (III) removal and recovery from Saccharomyces cerevisiae. Chemical Engineering Journal, 105(1-2), 11-20. https://doi.org/10.1016/j.cej.2004.07.009

Han, X., \& Gu, J. D. (2010). Sorption and transformation of toxic metals by microorganisms. Environmental Microbiology, 2nd ed.; Mitchell, R., Gu, JD, Eds, 153-176. R e c u p e r a d o d e : https://www.researchgate.net/profile/Xu_Han9 2/publication/230205674_Environmental_Micro biology_Second_Edition/links/5a392498aca272 10705d3253/Environmental-MicrobiologySecond-Edition.pdf

Igiri, E., Okoduwa, I., Idoko, O., Akabuogu, P., Adeyi, O., \& Ejiogu, K. (2018). Toxicity and bioremediation of heavy metals contaminated ecosystem from tannery wastewater: a review. Journal of toxicology, 2018 . https://doi.org/10.1155/2018/2568038
Irazusta, V., Bernal, A. R., Estévez, M. C., \& de Figueroa, L. I. (2018). Proteomic and enzymatic response under $\mathrm{Cr}(\mathrm{VI})$ overload in yeast isolated from textile-dye industry effluent. Ecotoxicology and environmental safety, 148, 490-500. https://doi.org/10.1016/j.ecoenv.2017.10.076

Ksheminska, H., Fedorovych, D., Babyak, L., Yanovych, D., Kaszycki, P., \& Koloczek, H. ( 2005). Chromium (III) and (VI) tolerance and bioaccumulation in yeast: a survey of cellular chromium content in selected strains of representative genera. Process Biochemistry, 40 ( 5 ), $1565-1572$. https://doi.org/10.1016/j.procbio $.2004 .05 .012 \mathrm{G}$ et rights and content

Macchi, M. (2018). Desarrollo de consorcios bacterianos con alta eficiencia de degradación de $\mathrm{PAH}$ para su aplicación a la recuperación de suelos crónicamente contaminados (Doctoral dissertation, Facultad de Ciencias Exactas). $\mathrm{R}$ e c u p e r a d o d e : http://sedici.unlp.edu.ar/handle/10915/66553

Maier, M., Pepper, L., \& Gerba, P. (2009) Environmental Microbiology ( $2^{\mathrm{a}}$ ed.) Academic Press. Elsevier, San Diego California. 585 pp. ISBN: 9780080919409

Malaviya, P., \& Singh, A. (2016). Bioremediation of chromium solutions and chromium containing wastewaters. Critical reviews in microbiology, 42 ( 4 ), $607-633$. https://doi.org/10.3109/1040841X.2014.97450 1

Marzan, W., Hossain, M., Mina, A., Akter, Y., \& Chowdhury, M. (2017). Isolation and biochemical characterization of heavy-metal resistant bacteria from tannery effluent in Chittagong city, Bangladesh: Bioremediation viewpoint. The Egyptian Journal of Aquatic Research, 43(1), 6574. https://doi.org/10.1016/j.ejar.2016.11.002

Mella, B., Glanert, C., \& Gutterres, M. (2015). Removal of chromium from tanning wastewater and its reuse. Process Safety and Environmental Protect i o n, $95,195-201$. https://doi.org/10.1016/j.psep.2015.03.007

Mishra, S., \& Bharagava, N. (2016). Toxic and genotoxic effects of hexavalent chromium in environment and its bioremediation strategies. Journal of Environmental Science and Health, Part C , $34(1), \quad 1-32$. c https://doi.org/10.1080/10590501.2015.10968 83 
Orbegozo, J., Abanto, M., García, R., \& Ramírez, P. (2008). Identificación molecular de Pichia guillermondii aislada de aguas ácidas de minas en el Perú y su resistencia a metales pesados. Revista Peruana de biología, 15(1), 91-95. R e c u p e r a d o d e : http://www.scielo.org.pe/scielo.php?pid $=\mathrm{S} 172$ 7-99332008000100013\&script=sci_arttext

Parveen, B., \& Singh, D. (2017). Assessment of Physico-Chemical Properties of Tannery Waste Water and Its Impact on Fresh Water Quality. International Journalof Current Microbiol. App. S c i , 6, $1879-1887$. D O I : https://doi.org/10.20546/ijcmas.2017.604.224

Rosales, C. (2018). Crecimiento dimórfico y caracterización molecular de Candida guillermondi aislado de Pannicum maximun (Doctoral dissertation, Escuela de Biología, Centro de Investigación en Biotecnología, Instituto Tecnológico de Costa Rica. Costa Rica). DOI: $10.18845 /$ tm.v31i1.3502

Rubio, I., Calderón, A., Gualtero, P., Acosta, R., \& Sandoval, J. (2015). Tratamientos para la remoción de metales pesados comúnmente presentes en aguas residuales industriales. Una revisión. Ingeniería y Región, (13), 73-90. R e c u p e r a d o d e : file:///C:/Users/USUARIO/Downloads/DialnetTratamientosParaLaRemocionDeMetalesPesados Comunmen-5432290\%20(2).pdf

Salari, R., \& Salari, R. (2017). Investigation of the best Saccharomyces cerevisiae growth condition. Electronic physician, 9(1), 3592. doi: $10.19082 / 3592$

Sibirny, A. A., \& Boretsky, Y. R. (2009). Pichia guilliermondii. In Yeast biotechnology: diversity and applications (pp. 113-134). Springer, Dordrecht. DOI: https://doi.org/10.1007/9781-4020-8292-4.
Siddiquee, S., Rovina, K., Azad, A., Naher, L., Suryani, S., \& Chaikaew, P. (2015). Heavy metal contaminants removal from wastewater using the potential filamentous fungi biomass: a review. J Microb Biochem Technol, 7(6), 384-395. DOI: 10.4172/1948-5948.1000243

Suárez, C., Garrido, N., \& Guevara, C. (2016). Levadura Saccharomyces cerevisiae y la producción de alcohol. Revisión bibliográfica. ICIDCA. Sobre los Derivados de la Caña de Azúcar, 50 ( 1 ). R e c u p e r a d o d e : https://www.redalyc.org/pdf/2231/2231484200 04.pdf

Tejada, C., Villabona, Á., \& Garcés, L. (2015). Adsorción de metales pesados en aguas residuales usando materiales de origen biológico. TecnoLógicas, 18(34), 109-123. Recuperado de: https://www.redalyc.org/pdf/3442/3442343360 10.pdf

Wang, J., \& Chen, C. (2009). Biosorbents for heavy metals removal and their future. Biotechnology advances, 27(2), 195-226. https://doi.org/10.1016/j.biotechadv.2008.11.0 02

Wu, Y., Mi, X., Jiang, L., Li, B., \& Feng, S. (2011). Equilibrium, kinetics and thermodynamics study on biosorption of $\mathrm{Cr}$ (VI) by fresh biomass of Saccharomyces cerevisiae. Korean Journal of Chemical Engineering, 28(3), 895-901. DOI: 10.1007/s11814-010-0429-7

Recibido: 15 mayo 2019| Aceptado: 20 julio 2019 